\title{
Improving Contact-Friction Conditions in Dynamic Simulations: Application to the Taylor Impact Test
}

\author{
J. Bonini, F. Llorca*, H. Bung and A. Juanicotena* \\ CEA Saclay, DRN/DMT/SEMT/DYN, Bâtiment 607, 91191 Gif-sur-Yvette, France \\ ${ }^{*}$ CEA Valduc, DAM/DRMN/HPC, 21120 Is-sur Tille, France
}

Abstract: The aim of this work is to test new contact-friction modelling by means of the elastoplastic Taylor impact-test. The contact-impact Lagrange multiplier method is developped in a dynamic explicit finite element frame. It allows the continuity of the contact conditions without the definition of any shock stiffness. This technique couples the calculation of the contact forces with all the other links of the mechanical system.

\section{Introduction}

The contact-impact modelling is an essential step in the prediction of the behaviour of materials and structures under shock loading. We only find in litterature [HAL 85, CAR 94] elastic impact-tests to validate new contact conditions, and no test about elastoplastic impact. The Taylor-rod impact-test is now a classical mean of validating the dynamic constitutive laws of metallic materials. The correlation between experimental and numerical results is established by comparison between the final shapes of the deformed projectile. Nevertheless, the impact loading is only represented by a boundary condition on one degree of freedom. The aim of this work is to test new contact-impact modelling by using the elastoplastic Taylor impact-test. The model is based on the Lagrange multiplier technique applied to a dynamic explicit integration scheme [BON 95].

\section{Numerical contact-impact modelling}

Three different kinds of contact exist for a finite element modelling (Fig.1). Node-onnode impact is used in case of compatible deformable mesh or rigid projectile. In case of deformable bodies with uncompatible mesh, the contact is defined between lines (2D) or between surfaces (3D). The contact modelling falls into two steps:

- the contact-searching: master-slave nodes technique is used.

- the calculation of a contact force to ensure the non-penetration of the target by the projectile. In case of dynamic finite elements codes, the contact force must be added to the the external forces in the fundamental equation:

$$
\begin{aligned}
& {[M]\left\{\gamma_{n+1}\right\}=\left[\left\{F_{n+1}^{\text {ext }}\right\}+\left\{F_{n+1}^{\text {contact }}\right\}\right]-\left\{F_{n+1}^{\text {int }}\right\}} \\
& {[M] \quad \text { mass matrix }} \\
& \left\{\gamma_{n+1}\right\} \text { acceleration vector at time step } n+1 \\
& \left\{F_{n+1}^{e x t}\right\} \quad \text { external forces vector } \\
& \left\{F_{n+1}^{\text {contact }}\right\} \text { interaction and contact forces vector } \\
& \left\{F_{n+1}^{i n t}\right\} \quad \text { internal forces vector }
\end{aligned}
$$


The penalty method [HAL 85] is widely used in dynamic explicit finite element codes to represent the impact conditions. The projectile and the target are linked by a spring of stiffness $\mathrm{k}$. This leads to necessary mesh-overlapping. The quality of the results is conditionned by the definition of a well-adapted value to $\mathrm{k}$. Moreover, the method uncouples on each node the calculation of the contact forces. The interaction due to the impact of more than one node on the same element cannot be represented.

The new contact-friction modelling, based on the Lagrange multiplier technique, allows a coupled calculation of all the nodes in contact to avoid mesh-overlapping, without any shock stiffness. Because of the integration scheme, the different links between the nodes of the mesh at time step $n+1$ can be written as a function of the acceleration $\left\{\gamma_{n+1}\right\}$ :

$$
\left[C_{n+1}\right]\left\{\gamma_{n+1}\right\}=\left\{D_{n+1}\right\}
$$

with $\left[C_{n+1}\right](\mathrm{m} \mathrm{x} \mathrm{n})$ matrix, $\mathrm{m}$ is the number of links, $\mathrm{n}$ the number of dof, $m \leq n$.

To solve the constrained system (1) and (2), we must find, as for the static solution, the Lagrange multiplier $\lambda$ which verifies:

$$
\begin{aligned}
& \min _{\gamma} \max _{\lambda}((L(\gamma, \lambda)) \\
& \text { with } \left.(L(\gamma, \lambda))=\frac{1}{2}\{\gamma\}^{t}[M]\{\gamma\}-\{\gamma\}^{t}\{F\}+\{\gamma\}^{t}[C]^{t}\{\lambda\}\right)
\end{aligned}
$$

The mass matrix $M$ is symmetrical, defined and positive, so can be inversed.

$$
\text { Let }[H]=\left[C_{n+1}\right][M]^{-1}\left[C_{n+1}\right]^{t}
$$

The $\mathrm{H}$ matrix is square $(\mathrm{m} \times \mathrm{m})$, symmetrical, positive, but only semi-defined. The $\mathrm{H}$ matrix can be inversed only if there is no redundant link. We show the existence of a solution $\left\{\lambda_{n+1}\right\}$ :

$$
\left\{\lambda_{n+1}\right\}=[H]^{-1}\left(\left\{D_{n+1}\right\}-\left[C_{n+1}\right][M]^{-1}\left[\left\{F_{n+1}^{e x t}\right\}-\left\{F_{n+1}^{i n t}\right\}\right]\right)
$$

The Coulomb's law is chosen to represent friction conditions. It consists in a condition on the tangential component of the velocity at the interface depending of the normal component and a friction coefficient $\mu$ :

$$
\left\|F_{t}\right\| \leq \mu\left\|F_{n}\right\| \quad \text { with } v_{t}=\left\{\begin{aligned}
0 & \text { si }\left\|F_{t}\right\|<\mu\left\|F_{n}\right\| \\
-c F_{t}(c>0) & \text { si }\left\|F_{t}\right\|=\mu\left\|F_{n}\right\|
\end{aligned}\right.
$$

The friction coefficient is calculated as a function of three parameters to take into account the decrease of friction when velocity increases:

$$
\mu=\mu_{\text {dynamic }}+\left(\mu_{\text {static }}-\mu_{\text {dynamic }}\right) e^{-\gamma V_{r}}
$$

$\mu_{\text {static }}$ and $\mu_{\text {dynamic }}=$ static and dynamic friction coefficient

$\gamma=$ decrease paramater of the exponential law 
$V_{r}=$ relative velocity of the two solids in contact

The Lagrange multiplier is calculated at time step $n+1$ to ensure:

$$
\left\{\begin{array}{l}
\left(v_{n+3 / 2}^{\text {slave }}\right)^{t}=\left(v_{n+3 / 2}^{\text {master }}\right)^{t} \\
\left(v_{n+3 / 2}^{\text {slave }}\right)^{n}=\left(v_{n+3 / 2}^{\text {master }}\right)^{n}
\end{array} \quad \text { so } \quad\left[C_{n+1}\right]\left\{v_{n+3 / 2}\right\}=0\right.
$$

- the equality of the velocities on the normal to the master surface at time step $\mathrm{n}+3 / 2$ for contact conditions. We shows that this condition leads to the equality of the displacements, velocities and accelerations at time step $n+2$. The normal contact forces are calculated as long as the loss of contact condition is not satisfied. This test consists in a condition of the sign of the Lagrange multiplier: a change of sign leads to a local loss of contact. The loss of contact can lead to an error on the other link forces calculated during the same time step. Nevertheless, due to the explicit integration scheme, we show that the error is negligible if time steps are small. Moreover, the new contact conditions are written and verified at the next time step.

- the equality of the velocities on the tangent at the master surface at time step $n+3 / 2$ for friction conditions. If the tangential force is greater than $\mu$ times the normal force, then the tangential force is projected on the friction cone. As previoulsy shown, the projection generates a negligible error on the other link forces.

\section{Application to the Taylor-impact test}

The specimen is $\operatorname{rod}(\mathrm{r}=4 \mathrm{~mm}, \mathrm{l}=20 \mathrm{~mm})$. This rod is placed into a polyvinyl support. The assembly support-rod is thrown at few hundred meter per second by a $16 \mathrm{~mm}$ calibre gaz-gun. The calibre of the gun is reduced to stop the support and let the rod freely flying to a rigid target in the shooting chamber (Fig.1). The velocity of the assembly is measured before the shock by means of an optical laser-sensor. The profile of the deformed specimen is taken after impact as shown on figure 2 for copper rods impacted at $107 \mathrm{~m} / \mathrm{s}$.

The studied material is represented by an elastoplastic constitutive relation where charachteristics depend on the strain rate (Symond's law) [JON 89]. The impact velocity is $107 \mathrm{~m} / \mathrm{s}$. Calculations are axisymmetrical. Four nodes massive elements with eight degrees of freedom and four integration points are used to represent both rods ( 160 elements) and the rigid body (only one element). Four results are shown. The first one is obtained by the classical representation (boundary condition on the axial displacement), the second one by a uncoupled technique, the third and the fourth one by the coupled Lagrange multiplier method respectively without and with friction $\left(\mu_{\text {static }}=0.05\right)$. The uncoupled technique gives poor results. The comparison between the classical representation and the Lagrange multiplier method are the same, except at the beginning of the contact where a rebound of the center of the rod is detected with the contact modelling. This proves that the contact law is correct, but the results are not in accordance with experimental observations: an error of $20 \%$ is noticed on the final radius of the rods. Nevertheless, the friction modellling gives exactly the final radius and length of the rods. 


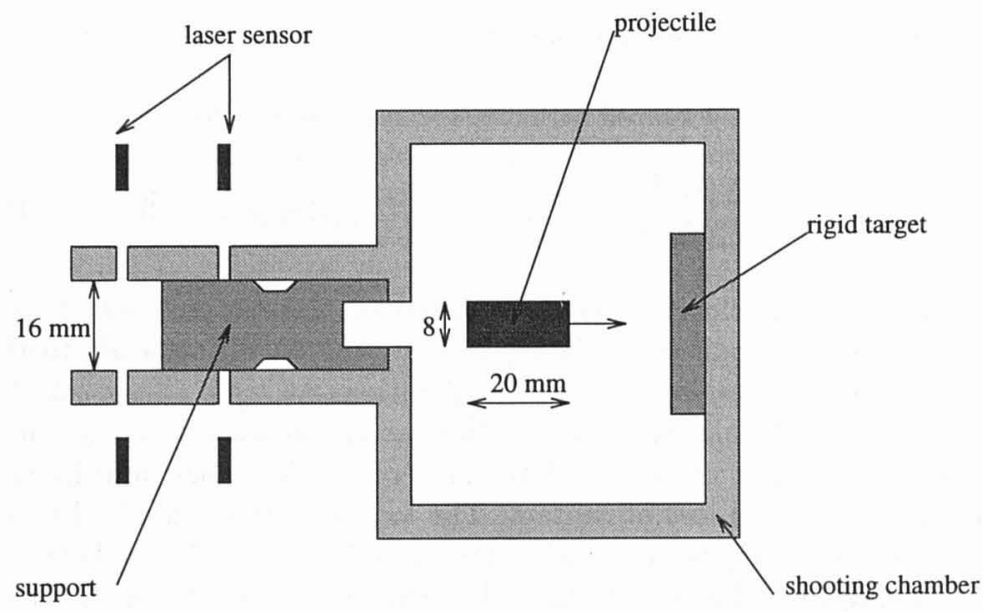

FIG. 1 - Taylor impact-test

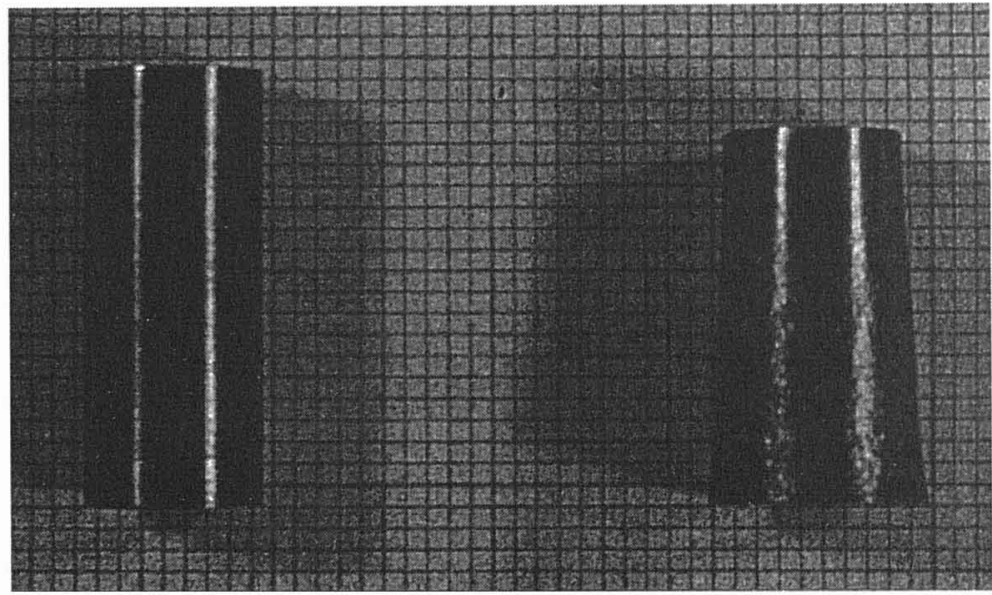

FIG. 2 - Copper-projectile before (left) and after (rigth) impact $(107 \mathrm{~m} / \mathrm{s}$ )

\begin{tabular}{|c|c|c|c|c|}
\hline & experiment & boundary cond. & Lagrange & Lagrange + friction \\
\hline final length & $16.68 \mathrm{~mm}$ & $16.52 \mathrm{~mm}$ & $16.54 \mathrm{~mm}$ & $16.65 \mathrm{~mm}$ \\
\hline final radius & $4.98 \mathrm{~mm}$ & $5.19 \mathrm{~mm}$ & $5.20 \mathrm{~mm}$ & $5 \mathrm{~mm}$ \\
\hline
\end{tabular}

ТАВ. 1 - Comparison of the final shape of the projectile 


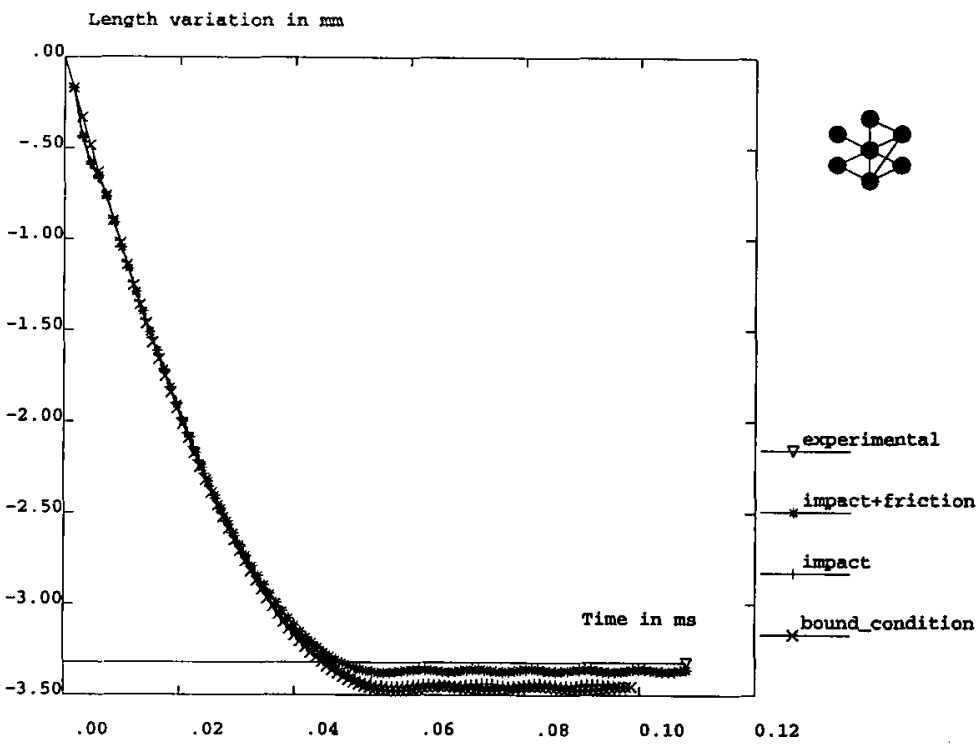

FIG. 3 - Taylor impact-test - projectile length vs. time

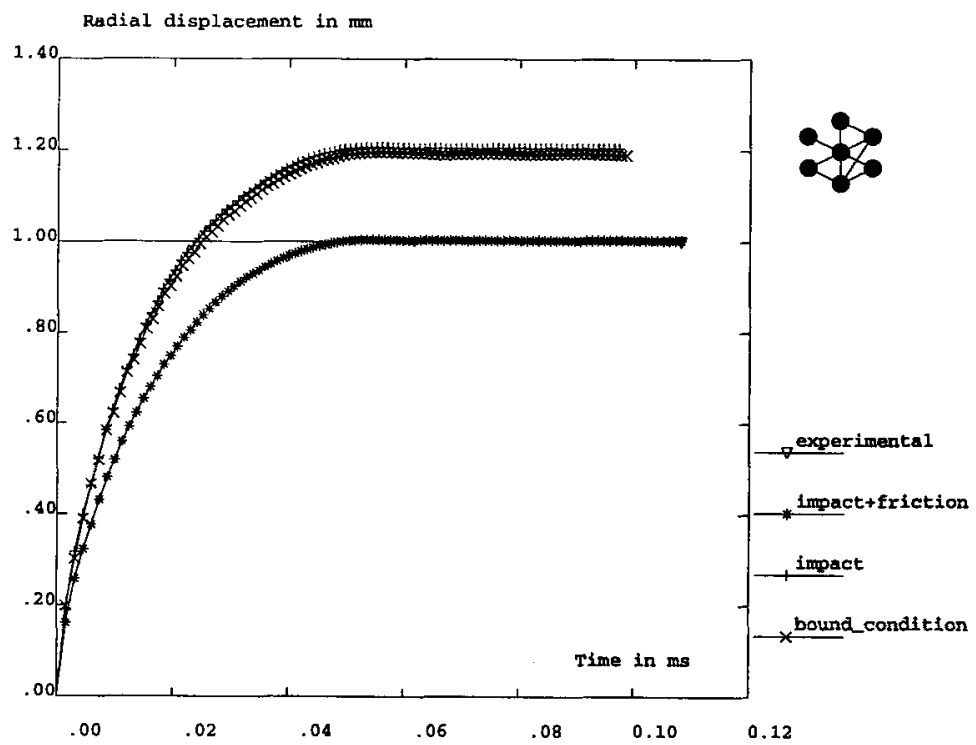

FIG. 4 - Taylor impact-test - projectile radius vs. time 


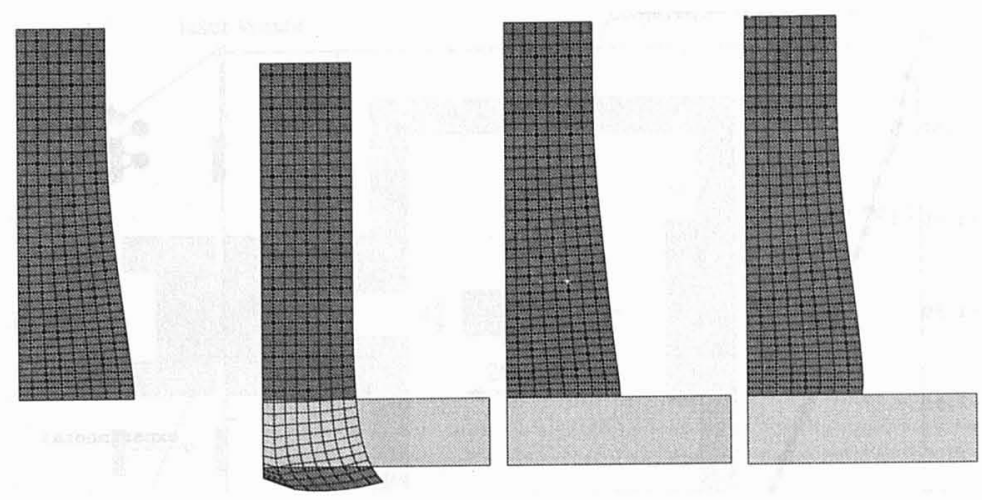

FIG. 5 - Taylor impact-test (left to right: boundary conditions - uncoupled - Lagrange multiplier - Lagrange multiplier with friction)

\section{Conclusion}

We show in this paper that the Taylor impact-test is well-adapted to validate contactimpact models. The contact-impact Lagrange multiplier method allows the continuity of the contact conditions without any condition as the definition of a shock stiffness. This technique couples the calculation of the contact forces with all the other links of the mechanical system. The cost of the assembly and the inversion at each time step of a link matrix is about $30 \%$ greater than an uncoupled method, but this method gives better results than the classical representation of the loading.

\section{Bibliography}

[BON95] Bonini J.,"Contribution à la prédiction numérique de l'endommagement de stratifiés composites sous impact basse vitesse", Thèse de Doctorat de Mécanique de l'Ecole Nat. Sup. des Arts et Métiers, 95-75-AM-09, 1995.

[HAL85] Hallquist J.O., Goudreau G.L., Benson D.J., "Sliding interfaces with contact-impact in large-scale Lagrangian computations", Comp. Meth. Appl. Mech. Eng., Vol.33, pp 107-137, 1985.

[HUG76] Hughes T.J.R., Taylor R.L., Sackman J.L., Curnier A., Kanoknulchai W., "A finite element method for a class of contact-impact problems", Comp. Meth. Appl. Mech. Eng., Vol.8, pp 249-276, 1976.

[JON89] Jones N., "Structural Impact", Cambridge University Press, 1989.

[LEP90] Lepareux M., Bung H., Matheron P., "Comportement des structures sous impact", Calcul des structures et Intelligence Artificielle, Vol.3, pp 31-52, Pluralis, 1990. 